\title{
Posiciones infundadas en la polémica sobre Mundos Posibles
}

\section{\ Joaquín Santiago Toranzo Calderón \\ BA-Logic, Lógica y Filosofía de la Lógica}

\begin{abstract}
Resumen
En la polémica entre actualistas y realistas modales, se ha recurrido a las nociones de necesidad y posibilidad lógicas, para responder a la pregunta por la existencia de los mundos posibles, defendiendo a partir de ellas sus contrapartidas metafísicas. Sin embargo, ¿pueden definirse unas puramente en términos de las otras? ¿O acaso es necesario algo más? En este trabajo se muestra que la relación entre estas nociones asume compromisos metafísicos previos recayendo en una justificación circular, por lo que no puede ser una opción viable para responder a tal pregunta.
\end{abstract}

\begin{abstract}
In the controversy between actualists and modal realist, the notions of logical necessity and possibility have been used to answer the question for the existence of possible worlds, defending from them their metaphysical counterparts. However, can ome be defined purely in terms of the others? Or maybe something else is needed? In this paper it is shown that the relation between these notions assumes previous metaphysical commitments falling into a circular justification, so it can not be a viable option to answer such a question.
\end{abstract}

\section{La Querella de los Mundos Posibles}

Es claro que en el uso cotidiano del lenguaje empleamos expresiones respecto a situaciones que podrían ser, o podrían haber sido, distintas a como de hecho son o fueron. Hay quienes defienden la idea de que estas expresiones refieren a mundos posibles, es decir, modos en que las cosas pueden ser o podrían haber sido. Pero eventualmente surge la pregunta: ¿esos mundos posibles, en qué sentido son posibles? ¿En el sentido de que no hay nada de contradictorio o inconsistente en ellos? ¿En el de que la realidad tiene alguna indeterminación que permite la existencia paralela de esos mundos? ¿O será que son descripciones posibles para una realidad sobre la que sólo tenemos una perspectiva incompleta?

Keywords

modal realism possible worlds logical necessity metaphysical necessity $\overline{\text { realismo modal }}$ mundos posibles necesidad lógica necesidad metafísica 
1. Para una introducción al actualismo y su discusión con el realismo modal referirse a Menzel (2014a) y Menzel (2016).

2. El término actual (y la familia de términos a la que pertenece) no tiene el uso español corriente para referirse a un tiempo presente, sino que es tomado del inglés y se corresponde (con cierta aproximación) al término español real. Tómese en cuenta para el resto de la discusión presentada, lo realmente efectivo no coincidirá necesariamente con lo actualmente existente, pero sí con lo efectivamente existente; por ello no se han presentado estas traducciones, sí disponibles en otros contextos.

3. Ver (Menzel, 2014b) para una presentación del realismo modal de Lewis en comparación con otras versiones disponibles.

4. Ambas consideraciones pueden encontrarse con distintos grados de desarrollo y énfasis en Lewis (1973: 84-91) y Lewis (1986).
Este trabajo abordará la polémica respecto a qué mundos son los que efectivamente existen, siendo las dos posturas más extremas las del realismo modal y la del actualis$m o,{ }^{1}$ donde la primera sostiene la existencia efectiva de todos los mundos posibles, mientras que la segunda niega que exista efectivamente algún mundo distinto del que habitamos (el mundo actual) ${ }^{2}$. Sin embargo, no tomaré partido por ninguna de estas posturas, sino que me limitaré a mostrar que ciertas estrategias de justificación para defender sus marcos metafísicos no pueden seguirse sin quedar infundadas.

Ambas posiciones asumen un principio común, el de que las cosas que existen efectivamente (dominio ontológico) lo hacen independientemente de las maneras en las que pueden ser referidas o descriptas (dominio lingüístico). Esto implica cierta preeminencia del dominio ontológico respecto del lingüístico. De esta manera, para el realista modal los enunciados sobre posibilidad refieren efectivamente a modos de ser de todos los mundos posibles que satisfagan esos enunciados. Por el contrario, para el actualismo este tipo de recursos lingüísticos no podrán referirse a entidades efectivamente existentes.

Aunque el realismo modal reúne a varias posiciones, tomaré la de David Lewis, un defensor fundamental con una propuesta original. ${ }^{3}$ Lewis ha sostenido con diversos argumentos la existencia efectiva de los mundos posibles. Dos de sus pilares se hallan en la intuición de que los modos en los que el mundo puede ser refieren a mundos tal como son, y al criterio por el cual de entre dos teorías debe preferirse la que recurra a menos tipos de entidades, la más "simple". ${ }^{4}$ A partir de ellos Lewis intenta mostrar que en realidad, el actualismo recurre a una teoría compleja que postula al menos dos tipos de mundos, los efectivos (el mundo actual) y los no efectivos (los mundos posibles), mientras que la suya recurre sólo a mundos efectivos. Su estrategia lo lleva a hacer un análisis del término actual como un término indexical (Lewis, 1970: 184-187) y a establecer que defender la existencia efectiva del mundo actual por su mero "privilegio" lingüístico no es una defensa aceptable, dado que se vuelve relativa al mundo desde el que se la defienda (Lewis, 1973: 85-86; 1986: 92-96).

Aunque la propuesta de Lewis ha sido puesta bajo sospecha por Eduardo Barrio (Barrio, 1999), Juan Di Leo (2016) ofrece una respuesta para rehabilitar el argumento realista. En su reconstrucción de la crítica de Barrio, se concentra en la dificultad que Barrio le adjudica a Lewis para distinguir "existir" de "existir actualmente", sea porque analiza la noción de posibilidad (junto a la de necesidad) a partir de los mundos efectivos para luego analizar la existencia efectiva a partir de la noción de posibilidad (justificación circular), sea porque supone que "existir" funciona como un cuantificador con rango sobre un dominio que excede lo actual (supone lo que pretende probar).

Dado que el realismo modal de Lewis requiere obtener satisfactoriamente tal distinción para recuperar su argumento realista, Di Leo intenta ofrecer un criterio distinto para diferenciar entre "existir" y "existir actualmente" que tampoco caiga en tales problemas. En este trabajo se muestra cómo es que el análisis de Di Leo cae en problemas semejantes a los de Lewis.

\section{Distinción Realista}

Antes de presentar los argumentos de Di Leo, es conveniente explicitar la semántica modal con la que trabaja. ${ }^{5}$ Para ello simplemente asumamos que tenemos un lenguaje al que le agregaremos dos operadores: posibilidad y necesidad, los cuales representaremos, respectivamente, con los símbolos $\diamond \mathrm{y} \square$, de manera que si $A$ es una fórmula de nuestro lenguaje $\diamond A$ y $\square A$ también lo son.
5. Mientras Di Leo adopta esta semántica Lewis ha llegado a una teoría que permita trabajar con sistemas alternativos (1968) para evitar el uso de una semántica modal. 
Una interpretación para la extensión del lenguaje consistirá en $\langle M, R, v>$, donde $M$ es un conjunto no vacío, cuyos elementos llamaremos mundos posibles, $R$ es una relación sobre pares ordenados de mundos posibles, a la que llamaremos relación de accesibilidad, y $v$ es una función que le asigna un valor de verdad a pares $\langle m, p\rangle$ (donde $m$ es un elemento de $M$ y $p$ es una proposición del lenguaje). La extensión del lenguaje consistirá en agregar cláusulas de interpretación para $\diamond \mathrm{y} \square$ :

$$
\begin{aligned}
& v_{m}(\diamond A)=1 \text { si, para algún } m^{*} \in M \text {, tal que } m R m^{*}, v_{m^{*}}(A)=1 \text {; y } 0 \text { en caso contrario. } \\
& v_{m}(\square A)=1 \text { si, para todo } m^{*} \in M \text {, tal que } m R m^{*}, v_{m^{*}}(A)=1 \text {; y } 0 \text { en caso contrario. }
\end{aligned}
$$

De esta manera, una expresión del tipo "Necesariamente p" será verdadera sólo si todos los mundos posibles a los que se accede desde el que se la ha emitido, valúan a $p$ como verdadera. Asimismo, una expresión del tipo "Posiblemente p" será verdadera sólo si algún mundo posible al que se accede desde el que se la ha emitido, valúa a $p$ como verdadera. Por lo tanto, el valor de verdad de las expresiones modales dependerá tanto del dominio de mundos posibles que se haya fijado como de la relación de accesibilidad que se haya estipulado. ${ }^{6}$

Ahora bien, según Di Leo, negar la distinción entre "existir" y "existir actualmente" nos compromete con restricciones en los dominios de cuantificaciones sobre mundos, restricciones que son problemáticas. Para comenzar, ofrece la diferencia entre

Necesariamente yo existo actualmente.

Necesariamente yo existo.

basándose en que las condiciones de verdad de una y otra no son equivalentes: mientras en la primera la cuantificación sobre mundos (dado el operador de necesidad) está restringida por el operador indexical de actualidad, en la segunda no hay restricciones de este tipo, pudiendo cuantificarse sobre todo el dominio. Por lo tanto, parecería que el valor de verdad de $E B$ depende del dominio, mientras que el de $E A$ no.

Tres argumentos son presentados para reforzar la intuición de que hay una asimetría entre $E A$ y $E B$. El primero de ellos (argumento modal) apela a las nociones intuitivas de necesidad y actualidad. El segundo (argumento ontológico) se presenta como independiente, pero pareciera que precisa al anterior, dado que recurre a un operador que capturaría la actualidad mostrando cómo es que se valúan las oraciones $E A$ y $E B$ :

$$
\mathrm{V}(@ \Phi)=1 \text { ssi } \quad \mathrm{V}(\Phi)=1 \text { en el mundo actual. }
$$

Finalmente, un tercer argumento (argumento epistémico) se centra en que $E A$ no requiere más información para ser valuada que su propia forma lógica, mientras que $E B$ necesita además información sobre el dominio ontológico. ${ }^{7}$

Ahora bien, los tres argumentos asumen parte de lo que se quiere probar, cayendo nuevamente en un círculo vicioso. Di Leo acusa al actualista con justa razón de que éste debe probar que el único mundo que existe efectivamente es el actual, y aún le resta dar una explicación para el uso de los términos modales en cualquier discurso. Sin embargo, así como el actualista se compromete con un dominio ontológico (con una estructura previa), Di Leo y Lewis caen en el mismo vicio.

En todos estos argumentos, la diferencia entre $E A$ y $E B$ depende de que la relación de accesibilidad entre mundos sea tal que permita que se acceda desde el mundo actual (definido en términos indexicales, es decir como el mundo que habita el emisor de
6. A la extensión modal de la Lógica Proposicional se la conoce como $\mathrm{K}$, presentada por primera vez en Kripke (1963). Sin embargo, he optado por una presentación más clara, perteneciente a Priest (2008: 21-23).
7. Si bien Di Leo no aclara específicamente qué información sobre el dominio ontológico es requerida, es de suponer que esta información está relacionada directamente con cuál es el dominio de mundos disponible y la relación de accesibilidad que hay entre ellos, principalmente la accesibilidad que goza el mundo desde el que se emiten ambas oraciones. 
estas oraciones) a algún mundo donde el emisor (o su contraparte si se quiere hablar en términos más lewisianos) no exista (Di Leo, 2016: 3-i pasos 3 y 4, 3-ii pasos 6 y 7). ¡Pero esto es exactamente lo que se acusó que el actualista no podía hacer sin dar una prueba independiente, prueba que Di Leo no ofrece!

Es cierto que la forma lógica de una y otra oración es distinta, y que su valor de verdad dependerá del alcance de los diferentes operadores. Pero no es su forma lógica lo que le interesa a Di Leo probar, sino que si $E B$ pudiera ser falsa, entonces querría decir que algo puede existir por fuera del mundo actual, siendo ello algo posible, pero efectivamente existente (algo no actual, pero existente).

\section{Versiones de lo posible y lo necesario}

Resta responder una última cuestión, la de si hay alguna manera legítima de defender una noción de posibilidad que coincida con una de necesidad, o más acorde a la discusión presentada, si hay una posición donde lo único que existe efectivamente sea lo que existe en el mundo actual, en el que habitamos. Para empezar es preciso separar los aspectos lógicos y metafísicos de ambas nociones.

La noción de posibilidad puede entenderse en términos de indeterminación (algo es posible porque aún no está determinado a ser de una manera u otra), de incompletitud (algo es posible porque es agregable a otras cosas), de ignorancia (algo es posible porque se desconoce si es imposible), de contingencia (algo es posible porque puede faltar), e incluso en términos de consistencia (algo es posible si al agregarlo no genera ningún conflicto, como incoherencias o trivialidades).

Necesidad es formulable en términos de determinación (algo es necesario porque está determinado a ser de una manera, pero no de otra), de requerimientos (algo es necesario porque hay algo que se da que no puede hacerlo si le falta esto), de esencia (algo es necesario porque no puede faltar, siempre está presente), y hasta de consecuencia o analiticidad (algo es necesario si se sigue de ciertas premisas o definiciones).

En un intento de capturar estas nociones, Saul Kripke (1963) desarrolló la semántica modal ya mencionada para el sistema $\mathbf{K}$, una extensión de la lógica proposicional clásica que incorpora dos operadores, uno para cada noción de nuestro interés. La semántica de tal sistema es ajustable para otras nociones fijando un dominio y una relación entre los elementos de ese dominio. Este dominio podrá tener mundos posibles u otras entidades que se considere que favorecerán la captura de las nociones de posibilidad y necesidad, como contextos incompletos o mundos imposibles. Las nociones capturadas dependerán entonces de lo que haya en el dominio y de la estructura interna que tenga, es decir, de cómo se relacionen sus elementos.

8. En Priest (2008: 46-49) pueden encontrarse una serie de argumentos (brevemente expuestos) sobre por qué aceptar o rechazar a S5 como el sistema que mejor captura la necesidad lógica, así como su relación con otro tipo de necesidades.
Se ha defendido extensamente ${ }^{8}$ que el sistema que mejor representa la noción de necesidad lógica es $\mathbf{5} 5$, donde el dominio sólo consta de mundos posibles que se relacionan todos universalmente con todos (la relación de accesibilidad es reflexiva, simétrica y transitiva). De esta manera lo único necesario es lo que afecta a la Lógica, mientras que el resto de los contenidos son posibles en igual medida. Colapsar la necesidad lógica de $\mathbf{S} 5$ con la necesidad metafísica implicaría que $E B$ es terminantemente falsa. Pero S5 no pretende capturar la necesidad metafísica sino sólo la lógica, la noción de que dadas las leyes de la Lógica hay ciertas oraciones que deben valer siempre, sin importar qué contenidos tenga cada mundo del dominio.

Imagínese ahora que se tiene un argumento, independiente a esta discusión, que concluye que todo estado de cosas, o mundo, es tal que es completo, es decir, que 
para toda oración, o bien ésta es verdadera, o bien falsa (o bien pertenece o bien no pertenece a este mundo). De esta manera, es argumentable que cuando se expresa una posibilidad nos estamos refiriendo con verdad o falsedad a este mismo mundo: cuando decimos que algo es posible y estamos diciendo algo verdadero es porque eso que es "posible" es de hecho así en este mundo; cuando decimos que algo es posible y no estamos diciendo algo verdadero, es porque este mundo es contradictorio con ello. Pero entonces, ¿cómo explicamos el discurso modal?

El actualista deberá recurrir, por ejemplo, a una teoría suplementaria donde al expresar falsas posibilidades (aquellas posibilidades que no coinciden con el mundo donde son emitidas) imaginamos que este mundo (a sabiendas o no) es de una manera que no es. Lo actual y lo verdaderamente posible coinciden, dado que todo lo que es verdaderamente posible es a la vez necesario. Por consiguiente, $E B$ sería verdadera.

El realista modal no parece necesitar de alguna teoría suplementaria sobre lo que podemos imaginar, dado que simplemente puede apelar a que las posibilidades que expresan diferencias con el mundo donde son emitidas simplemente refieren a otro mundo posible ("otro" dado que éste, el mundo actual es uno entre otros).

Otras opciones pueden presentarse para defender posiciones actualistas y realistas, pero no es mi interés defenderlas, sino mostrar que el intento de probar la existencia de los mundos posibles, así como su inexistencia, meramente desde las nociones lógicas de posibilidad y necesidad es insuficiente y requiere de tesis metafísicas independientes que en general ya contienen la respuesta al problema planteado, incluso previamente al análisis de estas nociones.

La confusión de que tales pruebas pueden darse, proviene probablemente del tratamiento que se haga de las formulaciones de las nociones de posibilidad y necesidad; es decir, surge de los supuestos previos que llevan a proponer un dominio de mundos y una relación de accesibilidad dados.

El sistema modal propuesto por Kripke, se limita a proponer las condiciones de verdad de las nociones mencionadas, permitiendo múltiples restricciones sobre el dominio de mundos o sobre la relación de accesibilidad. Sin embargo, cada una de estas restricciones, mientras sólo se hallen planteadas en términos lógicos, se dirigen a capturar nociones lógicas de posibilidad y necesidad (Menzel, 2014a: 3.3). Lo lógicamente posible y lo lógicamente necesario serán aquellas entidades que cumplan con las restricciones defendidas.

Ahora bien, si se ponen en la mesa relatos sobre los dominios de mundos o sobre las relaciones de accesibilidad, uno deja de hallarse sólo en el plano de la lógica. Por ejemplo, uno puede poner restricciones al dominio de mundos para que sólo lo integren los mundos que sean concebibles, y para ello recurrirá a una explicación de cuáles serán los mundos concebibles (e. g., aquellos que sean lógicamente posibles en un sistema modal dado y formulables por nuestra mente). Aún más, puede restringírselo a los mundos concebibles que no contradigan lo que se cree sobre el mundo desde el que se accede a ellos. Definitivamente estas interpretaciones van más allá de las nociones estrictamente lógicas de posibilidad y necesidad, sean interpretaciones metafísicas, epistémicas o de otro tipo, y parten de supuestos sobre sus nociones de posibilidad y necesidad correspondientes.

Este tipo de oscuridad en las nociones de posibilidad y necesidad permite caer en el error de hablar sobre sus versiones metafísicas, partiendo desde las definiciones de sus versiones lógicas. En el caso del actualista no parece ser un problema, dado que su interés en hacer coincidir lo actual con lo efectivamente existente no se defiende 
desde las versiones lógicas, sino desde otras (sean metafísicas, sean epistémicas, sean combinaciones). Pero el realista modal queda en la misma situación; tiene ahora la carga de la prueba respecto a por qué la relación de accesibilidad de las versiones metafísicas puede coincidir con la de las versiones lógicas o epistémicas.

\section{Conclusiones}

Dado que las nociones de posibilidad y necesidad pueden pertenecer a distintos planos filosóficos (como el lógico, el epistémico y el metafísico) existen distintas formulaciones para ellas. No he defendido especialmente ninguna relación de dependencia entre estas formulaciones; incluso si incurrí implícitamente en alguna, fue por motivos retóricos (por ejemplo, la dependencia o autonomía de las versiones metafísicas de las lógicas).

Di Leo requiere que haya una diferencia interesante entre las nociones de "existir" y "existir actualmente" para poder derivar de ella la pluralidad de mundos. Sin embargo, esta diferencia sólo puede ser interesante si se admite que están siendo comparadas sobre un plano equivalente, es decir que compartan un ámbito de interpretación en el que también puedan ser consideradas semejantes.

El realista modal partiría de la intuición de su diferencia, es decir que "existir" y "existir actualmente" no pueden emplearse equivalentemente; Di Leo apela especialmente a las distintas condiciones de verdad que tendrían las oraciones que las empleen. No obstante, cuando el actualista las interpreta como equivalentes, lo hace en un plano metafísico, empleando para ello las versiones metafísicas de las nociones de posibilidad y necesidad, en adición a los principios metafísicos propios de su postura. Si admitiera que no son equivalentes (junto al realista modal), será porque entiende que se ha pasado a emplear las versiones lógicas (o quizás tal vez las epistémicas), permitiéndole cuantificar sobre más mundos que el actual, mundos que no tomará como mundos efectivamente reales, sino de algún otro tipo. De esta manera, el actualista las puede considerar como equivalentes en un plano y como disímiles en otro.

Sin embargo, Di Leo no realiza las mismas comparaciones, dado que sus interpretaciones de "existir actualmente" siempre son interpretaciones metafísicas, mientras que a "existir" le asigna interpretaciones que no siempre son metafísicas. Por lo tanto, el análisis de Di Leo, o bien no ofrece una diferencia interesante entre las nociones pretendidas, o bien al hacerlo cae en los mismos problemas que Lewis al mezclar las versiones lógicas con las metafísicas de las nociones de posibilidad y necesidad (empleadas para trabajar con las de "existir" y "existir actualmente"). 


\section{Q Bibliografía}

" Barrio, E. (1999). "Indexicalidad y realismo modal”, Cuadernos de Filosofía, 45, pp. 49-68.

" Di Leo, J. M. (2016). "Actualidad, indexicalidad y estatus ontológico", Cuadernos de Filosofía 67-68, pp. 115-124.

》 Kripke, S. (1963). "Semantical Analysis of Modal Logic I. Normal Propositional Calculi", Zeitschrift fur mathematische Logik und Grundlagen der Mathematik, 9 (5-6), pp. 67-96.

»Lewis, D. (1968). "Counterpart Theory and Quantified Modal Logic", The Journal of Philosophy, 65 (5), pp. 113-126.

» Lewis, D. (1970). "Anselm and Actuality", Noûs, 4, pp. 175-188.

» Lewis, D. (1973). Counterfactuals, Blackwell Publishers, Oxford, 2001.

"Lewis, D. (1986). On the plurality of Worlds, Blackwell, Malden.

" Menzel, C. (2014a). "Actualism", Stanford Encyclopedia of Philosophy.

" Menzel, C. (2014b). "Classical Possibilism and Lewisian Possibilism", Stanford Encyclopedia of Philosophy.

" Menzel, C. (2016). "Possible Worlds", Stanford Encyclopedia of Philosophy.

"Priest, G. (2008). An Introduction to non-Classical Logic: From If to Is, Cambridge University Press, New York. 
